\title{
TEORES DE COMPOSTOS BIOATIVOS EM CAFÉS TORRADOS E MOÍDOS COMERCIAIS
}

Romilaine Mansano Nicolau de Souza, Gisele André Baptista Canuto, Rafael Carlos Eloy Dias e Marta de Toledo Benassi* Departamento de Ciência e Tecnologia de Alimentos, Universidade Estadual de Londrina, CP 6001, 86051-970 Londrina - PR, Brasil

Recebido em 7/7/09; aceito em 9/12/09; publicado na web em 24/3/10

\begin{abstract}
LEVELS OF BIOACTIVE COMPOUNDS IN COMMERCIAL ROASTED AND GROUND COFFEES. The amounts of nicotinic acid, trigonelline, 5-CQA, caffeine, kahweol and cafestol in 38 commercial roasted coffees ranged from 0.02 to 0.04 ; 0.22 to 0.96 ; 0.14 to $1.20 ; 1.00$ to $2.02 ; 0.10$ to 0.80 and 0.25 to $0.55 \mathrm{~g} / 100 \mathrm{~g}$, respectively. Evaluation of color and content of thermo-labile compounds indicated similarity in roasting degree. Differences in the levels of diterpenes and caffeine, components less influenced by the roasting degree, could be mainly explained by the species used (arabica and robusta). Gourmet coffees showed high concentrations of diterpenes, trigonelline and 5-CQA and low levels of caffeine, indicating high proportion of arabica coffee.
\end{abstract}

Keywords: caffeine; kahweol; cafestol.

\section{INTRODUÇÃO}

O café é uma das bebidas mais aceitas e apreciadas em diversos países no mundo, pelos aromas e sabores distintos e, mais recentemente, por seus potenciais efeitos benéficos na saúde humana. ${ }^{1}$ Dentre aproximadamente 100 espécies conhecidas do gênero Coffea as mais importantes economicamente no mercado internacional são Coffea arabica e Coffea canephora. ${ }^{2}$ No Brasil, a quase totalidade das lavouras de café $C$. canephora, genericamente conhecido por robusta, é da variedade conilon. ${ }^{3}$

As misturas de grãos (blends) são muito utilizadas quando se deseja manter a uniformidade nas características do produto. Nestes blends podem ser adicionados grãos de diferentes espécies, variedades e safras, tendo como objetivo a padronização do café. ${ }^{4} \mathrm{O}$ café conilon proporciona uma bebida de qualidade sensorial inferior quando comparado ao arábica.$^{5-8}$ Entretanto, é um produto atraente para misturas comerciais, pois diminui os custos e ajusta a bebida à preferência ou costume dos consumidores..$^{9,10}$

Para cafés torrados e moídos são descritas três categorias: Tradicional (arábica blendado com conilon até limite de 30\%), Superior (blend com até $15 \%$ de conilon) e Gourmet (somente arábica). Para essa classificação, além das diferentes proporções de café conilon, são sugeridas porcentagem máxima de defeitos e pontuação na análise sensorial..$^{9-12}$ Enquanto as designações Tradicional, Superior e Gourmet dizem respeito à qualidade, os termos clara, média e escura são empregados para indicar o grau de torra. ${ }^{9}$

Os cafés arábica e conilon podem ser caracterizados pelos teores de cafeína, trigonelina, ácido nicotínico, ácidos clorogênicos e alcoóis diterpênicos (caveol e cafestol). ${ }^{8,13,14} \mathrm{~A}$ influência desses compostos específicos na saúde tem sido relatada, destacando-se, além do valor nutricional (ácido nicotínico), sua correlação com a atividade antioxidante e a redução do risco de doenças crônicas degenerativas (cafeína, trigonelina e ácidos clorogênicos). Para diterpenos são descritos efeitos hepatoprotetor e anticarcinogênico, mas também a elevação da taxa de colesterol, atribuída ao cafestol. ${ }^{15}$ Estes compostos têm sido ainda estudados como ferramenta de discriminação das espécies, mas muitas vezes as concentrações variam, também, em função da origem geográfica e da intensidade da torração.,16-19

A cafeína é um alcaloide que apresenta relativa estabilidade ao processo térmico e contribui para o amargor da bebida. ${ }^{20,21} \mathrm{~A}$ trigo-

*e-mail: martatb@uel.br nelina contribui para o aroma do café pela formação de produtos de degradação durante a torração, principalmente o ácido nicotínico, que também é degradado em grau de torra intenso. ${ }^{1,17} \mathrm{O}$ ácido 5-cafeoilquínico (5-ACQ) é o representante majoritário do grupo dos ácidos clorogênicos, principais fenólicos encontrados no café, e é intensamente degradado durante a torração, originando pigmentos e voláteis aromáticos. ${ }^{7,21,22}$ Os diterpenos caveol e cafestol, encontrados somente no café, ${ }^{8}$ estão presentes na fração lipídica insaponificável e são pouco sensíveis ao processo de torração. ${ }^{16,17}$

No Brasil, maior produtor e exportador mundial de café, existem inúmeras marcas no mercado, produzidas por torrefadoras amplamente distribuídas no país. ${ }^{9}$ Entretanto, pouco se sabe sobre a composição desses produtos, principalmente tendo-se em vista que além de ser dependente da formulação dos grãos que compõem os blends, também apresenta variabilidade em função das condições de torração. ${ }^{23}$ Poucos trabalhos descrevem a composição de cafés comerciais de forma ampla, usualmente se atendo a alguma classe de compostos. A literatura descreve a caracterização em termos de somente ácidos clorogênicos, ${ }^{24}$ cafeína associada a ácidos clorogênicos, ${ }^{18,25}$ a trigonelina ${ }^{18,26} \mathrm{e}$, ainda, a ácido nicotínico e sacarose. ${ }^{26}$ Não existem dados relativos à composição de diterpenos.

Diante do exposto, o trabalho teve como objetivo caracterizar cafés torrados e moídos comercializados com diferentes denominações (Tradicional, Forte, Extra Forte, Gourmet, Premium, Aralto, Exportação e Espresso) quanto à cor e pela composição de ácido nicotínico, trigonelina, 5-ACQ, cafeína, caveol e cafestol.

\section{PARTE EXPERIMENTAL}

\section{Material}

Foram estudados cafés torrados e moídos comerciais (38 amostras), adquiridos no mercado ou cedidos por indústrias. Foram avaliadas 23 marcas, pertencentes a 13 fabricantes, indústrias de café associadas da ABIC (Associação Brasileira da Indústria de Café), que apresentavam em suas embalagens as seguintes denominações: Tradicional, Forte, Extra Forte, Extra Forte Clássico, Premium, Exportação, Aralto, Gourmet, Espresso. Para padronização da granulometria, as amostras foram passadas em peneira (ABNT 20). As amostras, acondicionadas em sacos plásticos, foram armazenadas em câmara fria a $10^{\circ} \mathrm{C}$ até o momento das análises. As determinações foram realizadas em duplicata, empregando-se um delineamento inteiramente ao acaso. 


\section{Caracterização das amostras}

Para análise de cor, as amostras de café foram acondicionadas em recipiente plástico de $1 \mathrm{~cm}$ de altura e $4 \mathrm{~cm}$ de diâmetro e foi empregado um colorímetro portátil Color-guide (BYK-Gardner, EUA), com geometria 45/0, iluminante D65 e área de leitura de $11 \mathrm{~mm}$ de diâmetro. ${ }^{27}$ O colorímetro forneceu diretamente os valores de $\mathrm{L}^{*}$ (luminosidade), $\mathrm{a}^{*}$ (componente vermelho-verde) e b* (componente amarelo-azul), e calculou-se o parâmetro tonalidade cromática $\left(\mathrm{H}^{*}=\operatorname{arctg} \mathrm{b}^{*} / \mathrm{a}^{*}\right)$.

A umidade foi determinada em equipamento de infravermelho (Ohaus-MB200, EUA) utilizando-se $105^{\circ} \mathrm{C}$, por 7 min e considerando-se $0,01 \mathrm{~g}$ como diferença de perda de peso. ${ }^{17}$ Os resultados, expressos em g/100 g, foram utilizados para o cálculo das concentrações dos constituintes químicos em base seca.

\section{Reagentes e padrões}

Foram utilizados como solventes para as etapas de extração e preparo da fase móvel: hidróxido de potássio (Vetec, Rio de Janeiro, Brasil) e tercbutil metil éter (Acrós Organics, New Jersey, EUA) de grau analítico; acetonitrila (J. T. Baker, EUA) e ácido acético glacial (J. T. Baker, EUA) de grau cromatográfico. A água empregada no preparo de padrões e soluções foi obtida por sistema de purificação e filtração Milli-Q ${ }^{\circledR}$ (Millipore, EUA). As fases móveis foram filtradas em sistema Millipore de filtração a vácuo utilizando-se membranas de celulose e nylon de 0,45 $\mu \mathrm{m}$ (Millipore, EUA) e degaseificadas antes de sua utilização (degaseificador Shimadzu DGU-14Avp, Kyoto, Japão).

Nas análises foram empregados padrões (grau analítico) de cafeína (1,3,7-trimetilxantina) (Acrós Organics, New Jersey, EUA), ácido nicotínico (n-metilnicotinamida) (Vetec, Rio de Janeiro, Brasil), ácido clorogênico (ácido 1,3,4,5-tetra-hidroxiciclo-hexanocarboxílico - 5-ACQ) e trigonelina (1-metilpiridinium-3-carboxilato mono-hidrato) da Sigma (Steinhelm, Alemanha). Foram também utilizados padrões de caveol e cafestol (Axxora, San Diego, EUA), mantidos em congelador $\left(-18^{\circ} \mathrm{C}\right)$.

\section{Equipamento}

Para as análises cromatográficas foi utilizado um cromatógrafo a líquido Shimadzu (Kyoto, Japão), constituído de um sistema de bombeamento de solventes com uma bomba quaternária (LC10ATvp) e degaseificador (DGU-14 Avp), válvula injetora Rheodyne, com alça de amostragem de $20 \mu \mathrm{L}$ e forno para a coluna cromatográfica (CTO-10 ASvp). O sistema estava acoplado a um detector espectrofotométrico UV/Visível de arranjo de diodos (DAD) Shimadzu (SPDM10Avp), varredura de 190 a $800 \mathrm{~nm}$ e sensibilidade de $0,8 \times 10^{-8} \mathrm{UA}$, conectado por uma interface (SCL-10Avp) a um microcomputador para processamento de dados.

\section{Análise cromatográfica}

Para determinação dos compostos hidrossolúveis (ácido nicotínico, trigonelina, 5-ACQ e cafeína), as amostras (0,5000 g) foram extraídas com acetonitrila:água $(5: 95, \mathrm{v} / \mathrm{v})$ a $80^{\circ} \mathrm{C}$ por $10 \mathrm{~min}$, e filtradas ( $7 \mathrm{~mm}$ ) em balão volumétrico de 100,0 mL. Dessa solução, uma alíquota $(5,0 \mathrm{~mL})$ foi transferida para balão volumétrico $(25,0$ $\mathrm{mL}$ ) completando-se o volume com a solução de extração. ${ }^{28}$

Para os compostos lipossolúveis (caveol e cafestol), as amostras $(0,2000 \mathrm{~g})$ foram saponificadas com 2,0 $\mathrm{mL}$ de hidróxido de potássio $2,5 \mathrm{~mol} / \mathrm{L}$ (em etanol $96 \%)\left(80^{\circ} \mathrm{C}, 1 \mathrm{~h}\right.$ ). Após adição de $2,0 \mathrm{~mL}$ de água destilada, foi feita extração dos insaponificáveis com $2,0 \mathrm{~mL}$ de tercbutil metil éter, agitação, centrifugação por $3 \mathrm{~min}$ a 3000 rpm, recolhendo-se a fase orgânica. O procedimento de extração foi repetido três vezes. Em seguida, foram adicionados $2,0 \mathrm{~mL}$ de água destilada ao extrato (para limpeza) e, após homogeneizar e descartar a fase aquosa, deixou-se evaporar próximo à secura a $70^{\circ} \mathrm{C}$. $\mathrm{O}$ extrato etéreo foi ressuspenso em 4,0 mL de fase móvel (acetonitrila $55 \%$ em água). ${ }^{29}$

Antes da injeção no cromatógrafo as amostras foram filtradas $(0,45 \mu \mathrm{m})$ (Millipore, EUA). Para a análise dos compostos hidrossolúveis, foram empregadas as condições cromatográficas descritas por Alves et al..$^{28}$ e para os lipossolúveis, a metodologia desenvolvida por Dias et al..$^{29}$ (Tabela 1).

Tabela 1. Condições cromatográficas empregadas na análise de compostos hidro e lipossolúveis

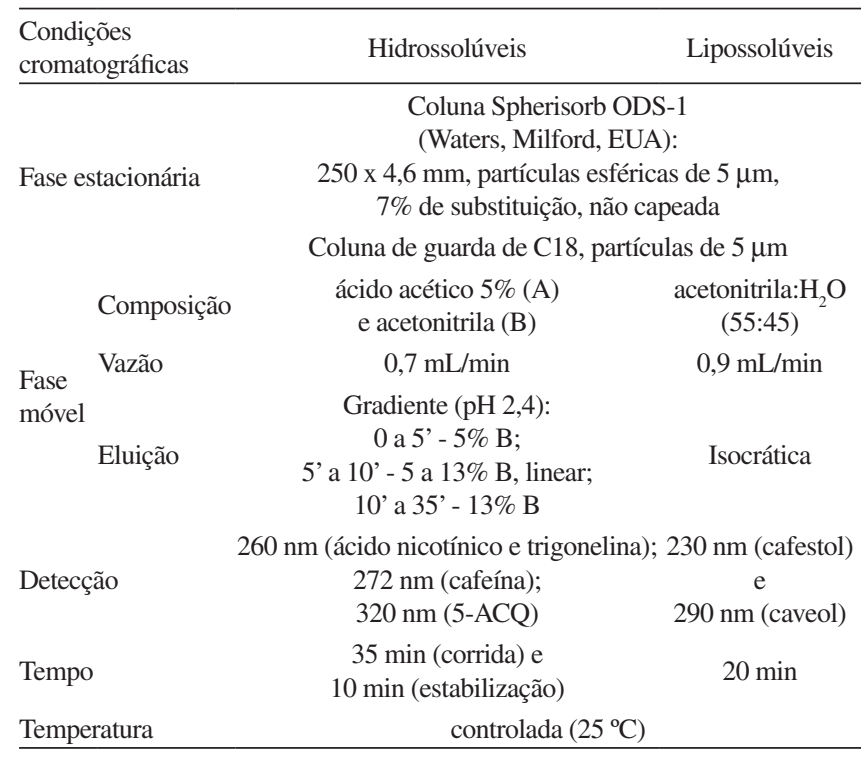

A identificação dos compostos foi feita no próprio cromatógrafo a líquido, com base nos tempos de retenção dos componentes eluídos da coluna comparados com o do padrão, pelo espectro obtido pelo detector (DAD) e empregando-se cocromatografia. A detecção dos compostos foi realizada em comprimentos de onda de máxima absorvância: ácido nicotínico e trigonelina (260 nm), cafeína (272 $\mathrm{nm})$, 5-ACQ $(320 \mathrm{~nm})$, cafestol $(230 \mathrm{~nm})$ e caveol $(290 \mathrm{~nm})$. A quantificação foi feita por padronização externa, construindo-se as curvas analíticas de calibração (com pelo menos cinco concentrações, em triplicata), onde a área do pico cromatográfico é proporcional à concentração de padrão injetado. As faixas de linearidade para os compostos analisados, baseadas em dados da literatura, ${ }^{16,28-30}$ foram de 0,01 a $0,10 \mathrm{~g} / 100 \mathrm{~g}$ para ácido nicotínico, 0,10 a 1,20 g/100 g para trigonelina, 0,02 a 2,20 g/100 g para 5-ACQ, 1,00 a 2,50 g/100 g para cafeína e 0,050 a 1,00 g/100 g para caveol e cafestol.

\section{Análise estatística}

Os dados foram submetidos à análise de variância (ANOVA), considerando-se a amostra ou denominação da embalagem do produto como causa de variação, e teste de Tukey $(\mathrm{p} \leq 0,05)$, utilizando-se o programa Statistica $7.1 .^{31}$

\section{RESULTADOS E DISCUSSÃO}

\section{Caracterização das amostras}

As amostras comerciais de café torrado e moído foram caracterizadas pelas determinações dos parâmetros de cor $\left(\mathrm{L}^{*}\right.$ e $\left.\mathrm{H}^{*}\right)$ e umidade (Tabela 2). 
Não houve diferença de umidade entre produtos com diferentes denominações, com os valores variando de 2,7 a 3,8 g/100 g de produto. Este intervalo encontra-se de acordo com o percentual máximo de umidade permitido em amostras comerciais $(5,0 \%)^{11}$ e dentro da faixa descrita por Campanha et al. ${ }^{16}$ e Dias, ${ }^{17}$ que considerando as diferentes espécies (arábica e conilon) e os diferentes graus de torra, relataram valores entre 1,2 a 4,0 g/100 g.

Tabela 2. Valores* de luminosidade, tonalidade cromática e umidade (g/100 g) para as diferentes denominações das amostras comerciais de café torrado e moído

\begin{tabular}{lccc}
\hline $\begin{array}{l}\text { Denominação } \\
\text { do produto** }\end{array}$ & Luminosidade $\left(\mathrm{L}^{*}\right)$ & $\begin{array}{c}\text { Tonalidade } \\
\text { cromática }\left(\mathrm{H}^{*}\right)\end{array}$ & Umidade \\
\hline Tradicional & $19,5^{\mathrm{a}} \pm 1,7$ & $54,1^{\mathrm{a}} \pm 2,4$ & $3,7^{\mathrm{a}} \pm 0,7$ \\
Forte & $21,2^{\mathrm{a}} \pm 1,4$ & $54,9^{\mathrm{a}} \pm 1,6$ & $3,8^{\mathrm{a}} \pm 0,7$ \\
Extra Forte & $20,5^{\mathrm{a}} \pm 1,8$ & $55,1^{\mathrm{a}} \pm 2,5$ & $3,7^{\mathrm{a}} \pm 0,7$ \\
Gourmet & $20,5^{\mathrm{a}} \pm 3,0$ & $55,3^{\mathrm{a}} \pm 3,6$ & $2,7^{\mathrm{a}} \pm 1,1$ \\
Premium & $19,3^{\mathrm{a}} \pm 0,3$ & $57,2^{\mathrm{a}} \pm 1,9$ & $2,9^{\mathrm{a}} \pm 0,7$ \\
Outras & $21,3^{\mathrm{a}} \pm 1,3$ & $54,5^{\mathrm{a}} \pm 2,9$ & $3,6^{\mathrm{a}} \pm 0,2$ \\
\hline
\end{tabular}

*Média \pm desvio padrão de 11 cafés (Tradicional), 4 (Forte), 13 (Extra Forte), 5 (Gourmet), 2 (Premium), 1 (Aralto), 1 (Exportação) e 1 (Espresso). Médias seguidas de uma mesma letra na coluna não diferem significativamente entre si (Tukey, $\mathrm{p} \leq 0,05)$. **Informação declarada na embalagem.

Ao caracterizar as amostras quanto à cor, não se observou diferença nos valores de $\mathrm{L}^{*}$ (de 19 a 21 ) e $\mathrm{H}^{*}$ (de 54 a 57), independentemente das informações diferenciadas, quanto ao grau de torra, na embalagem dos produtos (Tabelas 3 a 7). Fujioka e Shibamoto ${ }^{2.5}$ também não encontraram diferença de cor, avaliada pela absorvância no UV-Vis a $420 \mathrm{~nm}$, entre amostras de cafés comerciais.

Campanha et al. ${ }^{16}$ e Dias, ${ }^{17}$ estudando cafés arábica e conilon (puros e misturas) em diferentes graus de torra, observaram que os valores de $\mathrm{L}^{*}$ diminuíram com o aumento do grau de torra, apresentando faixas características que variaram entre 28 e 40 para a torra clara e para as torras média e escura estes valores estiveram na faixa de 16 a 30 e 13 a 21, respectivamente, em amostras puras. Os valores de $\mathrm{H}^{*}$ reportados nestes mesmos trabalhos variaram de 57 a 70 para torra clara, 45 a 65 para torra média e 41 a 59 para torra escura.

Dias ${ }^{17}$ descreveu ainda que, comparando-se amostras no mesmo grau de torra, as com maiores teores de conilon mostram-se mais amareladas (aumento no valor de $\mathrm{H}^{*}$ ) que as de arábica. Moura et $a l .{ }^{32}$ observaram também menor valor de $L^{*}$ (32) para o café arábica puro comparado ao conilon (34). Os blends (10 a 50\% de conilon) apresentaram valores intermediários, sendo que o aumento da porcentagem de conilon no blend elevou $\mathrm{L}^{*}$, clareando a amostra.

A comparação com dados de $\mathrm{L}^{*} \mathrm{e} \mathrm{H}^{*}$ da literatura demonstra que, para as 38 amostras comerciais analisadas neste estudo, há uma indicação de que foram submetidas à torra média ou escura, obedecendo ao padrão de preferência do consumidor brasileiro. ${ }^{33}$

\section{Quantificação dos compostos}

Os perfis cromatográficos típicos dos compostos hidro e lipossolúveis, nas amostras comerciais de café torrado e moído, podem ser observados nas Figuras $1 \mathrm{~S}$ e $2 \mathrm{~S}$ (material suplementar), respectivamente.

Os teores dos compostos analisados, encontrados nos cafés comerciais, estão apresentados nas Tabelas 3 a 7, de acordo com as diferentes denominações dos produtos. As marcas estão designadas pelas letras de A a W, e as denominações pelos códigos T (Tradicional), F (Forte), EF (Extra Forte), G (Gourmet), P (Premium), A (Aralto), EX (Exportação) e ES (Espresso). Apesar da variabilidade observada, todas as amostras apresentaram presença dos compostos bioativos (ácidos nicotínico e clorogênico, trigonelina, cafeína, caveol e cafestol).

Os teores de trigonelina variaram de forma expressiva, com algumas amostras apresentando teores quase cinco vezes superiores a outras (de 0,22 a 0,96 g/100 g do produto). Para ácido nicotínico, produto de degradação da trigonelina, observou-se variação de 0,021 a $0,038 \mathrm{~g} / 100 \mathrm{~g}$ do produto (Tabelas 3 a 7). Estes valores foram coincidentes aos relatados em cafés comerciais para trigonelina, de 0,20 a 0,96 g/100 g, ${ }^{18,26}$ e ácido nicotínico, de 0,010 e 0,030 g/100 g do produto. ${ }^{26}$

A literatura reporta que os teores de trigonelina variam de acordo com a espécie do grão, sendo que arábica apresenta maiores teores que conilon. Estudos sobre trigonelina no café verde apontam para valores entre 1,0 e 2,2\% de trigonelina em café arábica e entre 0,6 e 1,7\% em conilon. ${ }^{26,34,35}$ Estes teores diminuem após a torração em intensidade que depende do tempo e temperatura, gerando compostos voláteis e/ou não voláteis, ${ }^{7,36}$ destacando-se o ácido nicotínico, que também é degradado à medida que aumenta a intensidade do processo. ${ }^{17,37}$

Dias $^{17}$, trabalhando com misturas de cafés arábica e conilon em diferentes graus de torra, observou que com o aumento da proporção de conilon e da intensidade da torração, as concentrações de trigonelina e ácido nicotínico diminuíram, variando, respectivamente, de 0,93 g/100 g no arábica torra clara a 0,12 g/100 g no conilon torra escura,

Tabela 3. Teores* dos compostos hidro e lipossolúveis ( $\mathrm{g} / 100 \mathrm{~g}$ de amostra em base seca) em cafés denominados Tradicional (T)

\begin{tabular}{|c|c|c|c|c|c|c|c|}
\hline \multirow{2}{*}{\multicolumn{2}{|c|}{$\begin{array}{l}\text { Amostra / } \\
\text { Grau de torra** }\end{array}$}} & \multicolumn{4}{|c|}{ Compostos hidrossolúveis } & \multicolumn{2}{|c|}{ Compostos lipossolúveis } \\
\hline & & \multirow{2}{*}{$\frac{\text { Ác. Nicotínico }}{0,032^{\mathrm{ab}} \pm 0,00}$} & \multirow{2}{*}{$\frac{\text { Trigonelina }}{0,36^{\mathrm{ef}} \pm 0,00}$} & \multirow{2}{*}{$\frac{5-\mathrm{ACQ}}{0,25^{\mathrm{e}} \pm 0,01}$} & \multirow{2}{*}{$\frac{\text { Cafeína }}{1,11^{\mathrm{g}} \pm 0,01}$} & \multirow{2}{*}{$\frac{\text { Caveol }}{0,75^{\mathrm{a}} \pm 0,04}$} & \multirow{2}{*}{$\frac{\text { Cafestol }}{0,41^{\text {bc }} \pm 0,01}$} \\
\hline CT & média & & & & & & \\
\hline DT & média & $0,023^{\mathrm{c}} \pm 0,00$ & $0,56^{\mathrm{b}} \pm 0,02$ & $0,69^{\mathrm{a}} \pm 0,02$ & $1,66^{\mathrm{cd}} \pm 0,00$ & $0,41^{\mathrm{c}} \pm 0,01$ & $0,47^{\mathrm{a}} \pm 0,01$ \\
\hline ET & clássica & $0,033^{\mathrm{ab}} \pm 0,00$ & $0,34^{\mathrm{f}} \pm 0,00$ & $0,34^{\mathrm{cd}} \pm 0,01$ & $1,59^{\mathrm{de}} \pm 0,04$ & $0,40^{\mathrm{c}} \pm 0,00$ & $0,45^{\mathrm{ab}} \pm 0,02$ \\
\hline FT & & $0,031^{\mathrm{abc}} \pm 0,00$ & $0,24^{\mathrm{gh}} \pm 0,00$ & $0,21^{\mathrm{ef}} \pm 0,00$ & $1,58^{\mathrm{e}} \pm 0,01$ & $0,27^{\mathrm{d}} \pm 0,00$ & $0,35^{\mathrm{e}} \pm 0,01$ \\
\hline GT & & $0,034^{\mathrm{ab}} \pm 0,00$ & $0,26^{\mathrm{g}} \pm 0,00$ & $0,14^{\mathrm{g}} \pm 0,00$ & $1,25^{\mathrm{f}} \pm 0,00$ & $0,77^{\mathrm{a}} \pm 0,00$ & $0,47^{\mathrm{a}} \pm 0,01$ \\
\hline HT & média & $0,032^{\mathrm{ab}} \pm 0,00$ & $0,39^{\mathrm{e}} \pm 0,00$ & $0,38^{\mathrm{bc}} \pm 0,01$ & $1,63^{\text {cde }} \pm 0,03$ & $0,42^{\mathrm{c}} \pm 0,00$ & $0,40^{\mathrm{cd}} \pm 0,01$ \\
\hline KT & & $0,031^{\mathrm{abc}} \pm 0,00$ & $0,22^{\mathrm{h}} \pm 0,00$ & $0,18^{\mathrm{fg}} \pm 0,00$ & $1,79^{\mathrm{a}} \pm 0,02$ & $0,19^{\mathrm{e}} \pm 0,00$ & $0,36^{\mathrm{de}} \pm 0,01$ \\
\hline LT & & $0,027^{\mathrm{bc}} \pm 0,00$ & $0,53^{\mathrm{bc}} \pm 0,01$ & $0,42^{\mathrm{b}} \pm 0,00$ & $1,59^{\mathrm{de}} \pm 0,01$ & $0,49^{\mathrm{b}} \pm 0,01$ & $0,48^{\mathrm{a}} \pm 0,00$ \\
\hline MT & & $0,036^{\mathrm{a}} \pm 0,00$ & $0,50^{\mathrm{cd}} \pm 0,01$ & $0,68^{\mathrm{a}} \pm 0,03$ & $1,74^{\mathrm{ab}} \pm 0,02$ & $0,26^{\mathrm{d}} \pm 0,01$ & $0,40^{\mathrm{cd}} \pm 0,02$ \\
\hline QT & & $0,031^{\mathrm{abc}} \pm 0,00$ & $0,74^{a} \pm 0,03$ & $0,41^{\mathrm{b}} \pm 0,00$ & $1,07^{\mathrm{g}} \pm 0,01$ & $0,18^{\mathrm{e}} \pm 0,01$ & $0,26^{\mathrm{f}} \pm 0,01$ \\
\hline VT & média & $0,031^{\mathrm{abc}} \pm 0,00$ & $0,47^{\mathrm{d}} \pm 0,01$ & $0,33^{\mathrm{d}} \pm 0,00$ & $1,68^{\mathrm{bc}} \pm 0,03$ & $0,38^{\mathrm{c}} \pm 0,00$ & $0,38^{\text {cde }} \pm 0,01$ \\
\hline $\begin{array}{l}\text { MÉ } \\
(\mathrm{CV} \\
\end{array}$ & $\mathbf{A} \pm \mathbf{D P}$ & $\begin{array}{c}0,031 \pm 0,00 \\
(12)\end{array}$ & $\begin{array}{c}0,42 \pm 0,16 \\
(38)\end{array}$ & $\begin{array}{c}0,36 \pm 0,18 \\
(50)\end{array}$ & $\begin{array}{c}1,52 \pm 0,25 \\
(17)\end{array}$ & $\begin{array}{c}0,41 \pm 0,20 \\
(48)\end{array}$ & $\begin{array}{c}0,40 \pm 0,07 \\
(17)\end{array}$ \\
\hline
\end{tabular}

*Média de duas repetições \pm desvio padrão (DP). Letras distintas na mesma coluna indicam diferença significativa (Tukey, p $\leq 0,05)$. CV (\%): coeficiente de variação. **Informação especificada na embalagem do produto 
Tabela 4. Teores* dos compostos hidro e lipossolúveis ( $\mathrm{g} / 100 \mathrm{~g}$ de amostra em base seca) em cafés denominados Forte (F)

\begin{tabular}{|c|c|c|c|c|c|c|c|}
\hline \multirow{2}{*}{\multicolumn{2}{|c|}{$\begin{array}{l}\text { Amostra / } \\
\text { Grau de torra** }\end{array}$}} & \multicolumn{4}{|c|}{ Compostos hidrossolúveis } & \multicolumn{2}{|c|}{ Compostos lipossolúveis } \\
\hline & & \multirow{2}{*}{$\frac{\text { Ác. Nicotínico }}{0,029^{\mathrm{ab}} \pm 0,00}$} & \multirow{2}{*}{$\begin{array}{r}\text { Trigonelina } \\
0,42^{\mathrm{c}} \pm 0,02\end{array}$} & \multirow{2}{*}{$\frac{5-\mathrm{ACQ}}{0,40^{\mathrm{c}} \pm 0,02}$} & \multirow{2}{*}{$\frac{\text { Cafeína }}{1,57^{\mathrm{c}} \pm 0,01}$} & \multirow{2}{*}{$\frac{\text { Caveol }}{0,27^{\mathrm{g}} \pm 0,00}$} & \multirow{2}{*}{$\frac{\text { Cafestol }}{0,34^{\mathrm{h}} \pm 0,01}$} \\
\hline$\overline{\mathrm{AEF}}$ & & & & & & & \\
\hline DEF & escura & $0,026^{\mathrm{ab}} \pm 0,00$ & $0,41^{\mathrm{cd}} \pm 0,01$ & $0,46^{\mathrm{b}} \pm 0,00$ & $1,64^{\mathrm{bc}} \pm 0,05$ & $0,35^{\mathrm{e}} \pm 0,01$ & $0,44^{\text {de }} \pm 0,00$ \\
\hline $\mathrm{EEF}$ & Muito escura & $0,032^{\mathrm{ab}} \pm 0,00$ & $0,38^{\mathrm{cd}} \pm 0,00$ & $0,27^{\mathrm{fg}} \pm 0,00$ & $1,59^{\mathrm{c}} \pm 0,03$ & $0,41^{\mathrm{d}} \pm 0,01$ & $0,42^{\mathrm{ef}} \pm 0,02$ \\
\hline FEF & & $0,030^{\mathrm{ab}} \pm 0,00$ & $0,31^{\mathrm{e}} \pm 0,00$ & $0,25^{\mathrm{gh}} \pm 0,00$ & $1,59^{\mathrm{c}} \pm 0,00$ & $0,31^{\mathrm{f}} \pm 0,00$ & $0,36^{\mathrm{h}} \pm 0,00$ \\
\hline HEF & Média/escura & $0,036^{\mathrm{ab}} \pm 0,01$ & $0,38^{\mathrm{cd}} \pm 0,00$ & $0,40^{\mathrm{c}} \pm 0,00$ & $1,60^{c} \pm 0,09$ & $0,49^{\mathrm{c}} \pm 0,00$ & $0,44^{\text {cde }} \pm 0,01$ \\
\hline IEF & & $0,021^{\mathrm{b}} \pm 0,00$ & $0,61^{\mathrm{a}} \pm 0,03$ & $0,57^{\mathrm{a}} \pm 0,02$ & $1,58^{\mathrm{c}} \pm 0,04$ & $0,54^{\mathrm{b}} \pm 0,01$ & $0,49^{\mathrm{ab}} \pm 0,01$ \\
\hline JEF & & $0,026^{\mathrm{ab}} \pm 0,00$ & $0,53^{b} \pm 0,01$ & $0,54^{\mathrm{a}} \pm 0,01$ & $1,55^{\mathrm{c}} \pm 0,03$ & $0,43^{\mathrm{d}} \pm 0,02$ & $0,47^{\mathrm{abc}} \pm 0,01$ \\
\hline OEF & & $0,025^{\mathrm{ab}} \pm 0,00$ & $0,39^{\mathrm{cd}} \pm 0,02$ & $0,35^{\mathrm{d}} \pm 0,00$ & $2,02^{\mathrm{a}} \pm 0,01$ & $0,10^{\mathrm{h}} \pm 0,00$ & $0,37^{\mathrm{gh}} \pm 0,01$ \\
\hline QEF & & $0,028^{\mathrm{ab}} \pm 0,01$ & $0,62^{\mathrm{a}} \pm 0,01$ & $0,31^{\mathrm{e}} \pm 0,00$ & $1,05^{\mathrm{e}} \pm 0,00$ & $0,27^{\mathrm{g}} \pm 0,00$ & $0,34^{\mathrm{h}} \pm 0,00$ \\
\hline REF & & $0,038^{\mathrm{a}} \pm 0,01$ & $0,57^{\mathrm{ab}} \pm 0,00$ & $0,39^{\mathrm{c}} \pm 0,01$ & $1,19^{\mathrm{de}} \pm 0,02$ & $0,13^{\mathrm{h}} \pm 0,00$ & $0,25^{\mathrm{i}} \pm 0,01$ \\
\hline SEF & & $0,038^{\mathrm{a}} \pm 0,00$ & $0,36^{\mathrm{de}} \pm 0,02$ & $0,22^{\mathrm{h}} \pm 0,00$ & $1,27^{\mathrm{d}} \pm 0,05$ & $0,78^{\mathrm{a}} \pm 0,01$ & $0,49^{\mathrm{a}} \pm 0,01$ \\
\hline $\begin{array}{l}\text { SEF } \\
\text { clássico }\end{array}$ & & $0,033^{\mathrm{ab}} \pm 0,00$ & $0,57^{\mathrm{ab}} \pm 0,01$ & $0,31^{\mathrm{e}} \pm 0,01$ & $1,24^{\mathrm{d}} \pm 0,04$ & $0,76^{\mathrm{a}} \pm 0,00$ & $0,46^{\mathrm{bcd}} \pm 0,0 \mathrm{C}$ \\
\hline VEF & escura & $0,024^{\mathrm{ab}} \pm 0,00$ & $0,33^{\mathrm{e}} \pm 0,01$ & $0,30^{\mathrm{ef}} \pm 0,00$ & $1,76^{b} \pm 0,00$ & $0,37^{\mathrm{e}} \pm 0,01$ & $0,40^{\mathrm{fg}} \pm 0,00$ \\
\hline $\begin{array}{l}\text { MÉDIA } \\
\text { (CV\%) }\end{array}$ & & $\begin{array}{c}0,030 \pm 0,01 \\
(18)\end{array}$ & $\begin{array}{l}0,45 \pm 0,11 \\
(24)\end{array}$ & $\begin{array}{c}0,37 \pm 0,11 \\
(29)\end{array}$ & $\begin{array}{l}1,51 \pm 0,26 \\
(17)\end{array}$ & $\begin{array}{c}0,40 \pm 0,21 \\
(51)\end{array}$ & $\begin{array}{c}0,41 \pm 0,07 \\
(18)\end{array}$ \\
\hline
\end{tabular}

*Média de duas repetições \pm desvio padrão (DP). Letras distintas na mesma coluna indicam diferença significativa (Tukey, $\mathrm{p} \leq 0,05)$. CV (\%): coeficiente de variação.**Informação especificada na embalagem do produto.

Tabela 5. Teores* dos compostos hidro e lipossolúveis ( $\mathrm{g} / 100 \mathrm{~g}$ de amostra em base seca) em cafés denominados Extra Forte (EF)

\begin{tabular}{|c|c|c|c|c|c|c|c|}
\hline \multirow{2}{*}{$\begin{array}{l}\text { Amostra / } \\
\text { Grau de torra** }\end{array}$} & & \multicolumn{4}{|c|}{ Compostos hidrossolúveis } & \multicolumn{2}{|c|}{ Compostos lipossolúveis } \\
\hline & & Ác. Nicotínico & Trigonelina & 5-ACQ & Cafeína & Caveol & Cafestol \\
\hline $\mathrm{EF}$ & escura & $0,033^{\mathrm{a}} \pm 0,00$ & $0,36^{\mathrm{c}} \pm 0,01$ & $0,37^{\mathrm{b}} \pm 0,00$ & $1,57^{\mathrm{b}} \pm 0,00$ & $0,46^{\mathrm{b}} \pm 0,01$ & $0,45^{\mathrm{a}} \pm 0,00$ \\
\hline NF & média & $0,021^{\mathrm{b}} \pm 0,00$ & $0,63^{\mathrm{a}} \pm 0,01$ & $0,44^{\mathrm{a}} \pm 0,00$ & $1,62^{\mathrm{b}} \pm 0,03$ & $0,46^{\mathrm{b}} \pm 0,02$ & $0,46^{\mathrm{a}} \pm 0,03$ \\
\hline $\mathrm{PF}$ & & $0,032^{\mathrm{ab}} \pm 0,00$ & $0,37^{\mathrm{c}} \pm 0,02$ & $0,30^{\mathrm{c}} \pm 0,01$ & $1,75^{\mathrm{a}} \pm 0,04$ & $0,38^{\mathrm{c}} \pm 0,01$ & $0,40^{\mathrm{a}} \pm 0,00$ \\
\hline $\mathrm{TF}$ & & $0,033^{\mathrm{a}} \pm 0,00$ & $0,55^{\mathrm{b}} \pm 0,01$ & $0,31^{\mathrm{c}} \pm 0,01$ & $1,29^{\mathrm{c}} \pm 0,01$ & $0,60^{\mathrm{a}} \pm 0,02$ & $0,41^{\mathrm{a}} \pm 0,02$ \\
\hline $\begin{array}{l}\text { MÉDIA } \pm \text { DP } \\
(\text { CV\% })\end{array}$ & & $\begin{array}{c}0,030 \pm 0,01 \\
\text { (19) }\end{array}$ & $\begin{array}{l}0,48 \pm 0,13 \\
\quad(28)\end{array}$ & $\begin{array}{c}0,35 \pm 0,06 \\
\quad(18)\end{array}$ & $\begin{array}{l}1,56 \pm 0,19 \\
\quad(12)\end{array}$ & $\begin{array}{l}0,47 \pm 0,09 \\
\quad(20)\end{array}$ & $\begin{array}{c}0,43 \pm 0,03 \\
(6)\end{array}$ \\
\hline
\end{tabular}

*Média de duas repetições \pm desvio padrão (DP). Letras distintas na mesma coluna indicam diferença significativa (Tukey, $\mathrm{p} \leq 0,05)$. CV (\%): coeficiente de variação. **Informação especificada na embalagem do produto.

Tabela 6. Teores* dos compostos hidro e lipossolúveis (g/100 g de amostra em base seca) em cafés denominados Gourmet (G)

\begin{tabular}{|c|c|c|c|c|c|c|}
\hline \multirow{2}{*}{$\begin{array}{l}\text { Amostra / } \\
\text { Grau de torra** }\end{array}$} & \multicolumn{4}{|c|}{ Compostos hidrossolúveis } & \multicolumn{2}{|c|}{ Compostos lipossolúveis } \\
\hline & Ác. Nicotínico & Trigonelina & 5-ACQ & Cafeína & Caveol & Cafestol \\
\hline$\overline{\mathrm{BG}}$ & $0,032^{\mathrm{a}} \pm 0,00$ & $0,54^{\mathrm{c}} \pm 0,02$ & $0,60^{\mathrm{d}} \pm 0,03$ & $0,99^{\mathrm{b}} \pm 0,05$ & $0,74^{\mathrm{a}} \pm 0,03$ & $0,47^{\mathrm{a}} \pm 0,01$ \\
\hline clara & $0,032^{\mathrm{a}} \pm 0,00$ & $0,96^{\mathrm{a}} \pm 0,01$ & $1,20^{\mathrm{a}} \pm 0,00$ & $1,17^{\mathrm{a}} \pm 0,01$ & $0,77^{\mathrm{a}} \pm 0,03$ & $0,47^{\mathrm{a}} \pm 0,02$ \\
\hline FG & $0,029^{\mathrm{a}} \pm 0,00$ & $0,70^{\mathrm{b}} \pm 0,01$ & $0,72^{\mathrm{c}} \pm 0,03$ & $1,28^{\mathrm{a}} \pm 0,06$ & $0,57^{\mathrm{b}} \pm 0,01$ & $0,47^{\mathrm{a}} \pm 0,01$ \\
\hline média & $0,028^{\mathrm{a}} \pm 0,00$ & $0,88^{\mathrm{a}} \pm 0,04$ & $1,01^{\mathrm{b}} \pm 0,02$ & $1,23^{\mathrm{a}} \pm 0,01$ & $0,71^{\mathrm{a}} \pm 0,04$ & $0,44^{\mathrm{a}} \pm 0,02$ \\
\hline UG & $0,024^{\mathrm{a}} \pm 0,00$ & $0,38^{\mathrm{d}} \pm 0,01$ & $0,27^{\mathrm{e}} \pm 0,00$ & $1,29^{\mathrm{a}} \pm 0,00$ & $0,80^{\mathrm{a}} \pm 0,02$ & $0,46^{\mathrm{a}} \pm 0,01$ \\
\hline $\begin{array}{l}\text { MÉDIA } \pm \text { DP } \\
(\text { CV\% })\end{array}$ & $\begin{array}{c}0,029 \pm 0,00 \\
(11)\end{array}$ & $\begin{array}{c}0,69 \pm 0,24 \\
(34)\end{array}$ & $\begin{array}{c}0,76 \pm 0,36 \\
(48)\end{array}$ & $\begin{array}{c}1,19 \pm 0,12 \\
(10)\end{array}$ & $\begin{array}{c}0,72 \pm 0,09 \\
(13)\end{array}$ & $\begin{array}{c}0,46 \pm 0,01 \\
\text { (3) }\end{array}$ \\
\hline
\end{tabular}

*Média de duas repetições \pm desvio padrão (DP). Letras distintas na mesma coluna indicam diferença significativa (Tukey, $\mathrm{p} \leq 0,05)$. CV (\%): coeficiente de variação. **Informação especificada na embalagem do produto.

Tabela 7. Teores* dos compostos hidro e lipossolúveis (g/100 g de amostra em base seca) em cafés denominados Premium (P), Aralto (A), Exportação (EX) e Espresso (ES)

\begin{tabular}{|c|c|c|c|c|c|c|c|}
\hline \multirow{2}{*}{\multicolumn{2}{|c|}{$\begin{array}{l}\text { Amostra / } \\
\text { Grau de torra** }\end{array}$}} & \multicolumn{4}{|c|}{ Compostos hidrossolúveis } & \multicolumn{2}{|c|}{ Compostos lipossolúveis } \\
\hline & & Ác. Nicotínico & \multirow{2}{*}{$\begin{array}{c}\text { Trigonelina } \\
0,53^{\mathrm{b}} \pm 0,03\end{array}$} & \multirow{2}{*}{$\frac{5-\mathrm{ACQ}}{0,45^{\mathrm{c}} \pm 0,00}$} & \multirow{2}{*}{$\frac{\text { Cafeína }}{1,34^{\mathrm{c}} \pm 0,02}$} & \multirow{2}{*}{$\begin{array}{c}\text { Caveol } \\
0,49^{\mathrm{c}} \pm 0,02\end{array}$} & \multirow{2}{*}{$\frac{\text { Cafestol }}{0,44^{\mathrm{b}} \pm 0,02}$} \\
\hline $\mathrm{BP}$ & & $0,031^{\mathrm{a}} \pm 0,00$ & & & & & \\
\hline HP & média/escura & $0,033^{\mathrm{a}} \pm 0,00$ & $0,43^{\mathrm{c}} \pm 0,02$ & $0,38^{\mathrm{d}} \pm 0,00$ & $1,59^{\mathrm{b}} \pm 0,01$ & $0,57^{\mathrm{b}} \pm 0,01$ & $0,48^{\mathrm{b}} \pm 0,01$ \\
\hline DA & média & $0,032^{\mathrm{a}} \pm 0,00$ & $0,67^{\mathrm{a}} \pm 0,03$ & $0,60^{\mathrm{a}} \pm 0,01$ & $1,09^{\mathrm{d}} \pm 0,06$ & $0,67^{\mathrm{a}} \pm 0,02$ & $0,55^{\mathrm{a}} \pm 0,01$ \\
\hline DEX & média/clara & $0,024^{\mathrm{ab}} \pm 0,00$ & $0,48^{\mathrm{bc}} \pm 0,02$ & $0,57^{b} \pm 0,00$ & $1,43^{\mathrm{c}} \pm 0,03$ & $0,32^{\mathrm{e}} \pm 0,01$ & $0,44^{b} \pm 0,02$ \\
\hline WES & & $0,021^{\mathrm{b}} \pm 0,00$ & $0,62^{\mathrm{a}} \pm 0,00$ & $0,46^{\mathrm{c}} \pm 0,00$ & $1,73^{\mathrm{a}} \pm 0,01$ & $0,41^{\mathrm{d}} \pm 0,02$ & $0,45^{\mathrm{b}} \pm 0,01$ \\
\hline
\end{tabular}

*Média de duas repetições \pm desvio padrão. Letras distintas na mesma coluna indicam diferença significativa (Tukey, $\mathrm{p} \leq 0,05)$. **Informação especificada na embalagem do produto. 
e de $0,091 \mathrm{~g} / 100 \mathrm{~g}$ no arábica torra clara até a ausência no conilon torra escura. $\mathrm{O}$ autor concluiu ainda que estes compostos foram mais sensíveis à degradação na matriz de café conilon. Casal, Oliveira e Ferreira ${ }^{37}$ observaram comportamento semelhante para trigonelina, relatando que em café arábica houve variação de 0,89 a $0,05 \mathrm{~g} / 100 \mathrm{~g}$, do café verde ao mais torrado $\left(240^{\circ} \mathrm{C}\right)$ e para o café conilon, a variação foi de 0,63 a $0,10 \mathrm{~g} / 100 \mathrm{~g}$, para as mesmas condições, enquanto observaram comportamento diferenciado para o ácido nicotínico e relataram que a máxima concentração foi de $0,017 \mathrm{~g} / 100 \mathrm{~g}$ para o arábica e $0,013 \mathrm{~g} / 100 \mathrm{~g}$ para o conilon.

Assim, a variabilidade observada nos teores de trigonelina e ácido nicotínico nos produtos comerciais poderia ser atribuída tanto à espécie do grão empregado quanto ao processo. No entanto, considerando-se a similaridade nas cores das amostras (Tabela 2), que indica processo de torração semelhante, é provável que o processo tenha menos influência na concentração desses compostos do que a matéria-prima.

O 5-ACQ foi encontrado nas amostras em teores variando em torno de dez vezes, entre 0,14 e 1,20 g/100 g do produto (Tabelas 3 a 7). Estes valores foram semelhantes aos da literatura que menciona alteração no teor de 5-ACQ conforme a espécie e origem de café e durante a torração. Em amostras comerciais de café torrado e moído foram relatados teores de $0,11 \mathrm{a} 0,71 \mathrm{~g} / 100 \mathrm{~g}$ para o 5 -ACQ. ${ }^{18,25} \mathrm{Con}$ forme a variação no grau de torra (de escura a clara), foram descritos teores de 5-ACQ de 0,09 a 2,17 g/100 g para cafés arábica, e de 0,08 a $3,18 \mathrm{~g} / 100 \mathrm{~g}$ para conilon. ${ }^{17,24,35} \mathrm{O}$ café conilon é descrito, usualmente, por apresentar concentração mais elevada desse composto no grão verde. ${ }^{5,24}$ No entanto, Dias $^{17}$ e Trugo e Macrae ${ }^{38}$ relatam que o 5-ACQ apresenta maior susceptibilidade à temperatura na matriz de conilon; assim, em graus de torra mais intensos observa-se maior teor de 5-ACQ para café arábica. Desta forma, para 5-ACQ, tanto o processo de torração quanto a matéria-prima empregadas devem ter contribuído para a grande variação observada nos produtos de mercado.

Os cafés apresentaram teores de cafeína variando de 1,00 a 2,02 g/100 g do produto (Tabelas 3 a 7). A literatura relata que a cafeína é relativamente estável à temperatura e seus teores variam de acordo com a espécie, sendo que grãos de conilon apresentam maiores teores quando comparados aos de arábica. Foram descritos, para cafés de diferentes origens e graus de torra, teores de 0,88 a 1,68 g/100 g para café arábica, e de 1,57 a 2,68 g/100 g para conilon. ${ }^{17,28,35,39}$ Para amostras comerciais de café torrado e moído são relatados teores de 0,80 a 1,65 g/100 g. . $^{18,25,26}$ Observou-se que, no presente estudo, as amostras que apresentaram os maiores teores de cafeína estiveram acima da faixa usualmente descrita para arábica. Esse fato, associado à reconhecida estabilidade do composto à torração, permite atribuir a grande variabilidade dos teores de cafeína nos produtos de mercado principalmente à matéria-prima utilizada, provavelmente devido aos blends das espécies. No entanto, deve ser considerado que mesmo dentro da mesma espécie é possível obter-se variabilidade considerável. Para 17 diferentes cultivares brasileiros de arábica e híbridos, com diferentes métodos de preparo, foram relatados para café verde teores de 1,05 a 1,54 g/100 g. ${ }^{30}$

Com relação aos diterpenos, os teores ( $\mathrm{g} / 100 \mathrm{~g}$ do produto) encontrados nas amostras estiveram na faixa de 0,10 a 0,80 para caveol e de 0,25 a 0,55 para cafestol. Na literatura, não foram encontrados dados de diterpenos em cafés torrados e moídos comerciais reportados para comparação. Para o café arábica, são descritos teores de caveol e cafestol entre 0,10 e $0,87 \mathrm{~g} / 100 \mathrm{~g}$ e entre 0,10 e $0,70 \mathrm{~g} / 100$ $\mathrm{g}$ de café torrado, respectivamente. ${ }^{14,16,40-43}$ Para o café conilon, que possui menor teor de lipídios, é reportada concentração de cafestol na faixa de 0,08 a $0,37 \mathrm{~g} / 100 \mathrm{~g}$ de café torrado. ${ }^{14,16,41,42}$ Existe, ainda, controvérsia quanto à presença de caveol em café conilon. Alguns pesquisadores não observaram a presença deste composto, ${ }^{16,17,44}$ enquanto outros descrevem concentrações inferiores a 0,013 g/100 g de café torrado ${ }^{41}$ ou apenas traços..$^{14,42}$
Com a torração, ocorre uma elevação proporcional nos teores de lipídios totais de 11,4 para 15,4\% no café arábica e de 6,1 para $9,6 \%$ no café conilon, devido à destruição dos carboidratos. ${ }^{8,45}$ Os diterpenos caveol e cafestol, constituintes da matéria insaponificável, são relativamente estáveis à temperatura, sendo pouco degradados durante a torração do café. ${ }^{16,43}$ Assim, poderia haver um pequeno aumento proporcional em suas concentrações com a intensidade de processo. Desta forma, a variação dos teores de caveol e cafestol nas amostras pode estar relacionada com a matéria-prima, pois grãos de arábica apresentaram maiores teores de diterpenos, principalmente caveol, quando comparados aos grãos de conilon.

Considerando a variabilidade entre as diferentes denominações dos produtos (Tabelas de 3 a 7) verificou-se que ácido nicotínico e cafestol foram os componentes cujos teores apresentaram menor diferença entre as denominações, comparando-se os valores médios de concentração (de 0,021 a 0,032 g/100 g para ácido nicotínico e de 0,40 a $0,55 \mathrm{~g} / 100 \mathrm{~g}$ para cafestol), e, no geral, menor variabilidade dentro de cada denominação (CV \%).

Do ponto de vista de qualidade, os produtos Gourmet (Tabela 6) apresentaram os maiores valores médios de caveol, 5-ACQ e trigonelina $(0,72,0,76$ e $0,69 \mathrm{~g} / 100 \mathrm{~g}$, respectivamente), e o menor valor de cafeína (1,19 g/ 100 g). O café denominado Aralto (DA) (Tabela 7) apresentou composição semelhante. Observou-se, ainda, para os cafés Gourmet as menores variações intracategoria (CV \%) para os teores de compostos estáveis termicamente, como cafeína (10\%), caveol (13\%) e cafestol (3\%), indicando maior uniformidade na matéria-prima utilizada. Esse comportamento é condizente com o previsto pela legislação, ${ }^{11}$ tendo em vista que o café Gourmet deveria ser feito unicamente com grãos arábica, e o Tradicional poderia ter até $30 \%$ de café conilon. Isso justificaria não apenas os menores teores médios de caveol, 5-ACQ e trigonelina, e os maiores de cafeína para estes produtos, como a maior variabilidade (Tabela 3), tendo em vista que cada marca pode adotar um blend diferenciado. Dentro da denominação Tradicional, encontram-se desde cafés com teor de caveol (composto estável e só presente em café arábica) de 0,18 g/100 g (QT) até 0,75 g/100 g (CT), mostrando que a variabilidade de matéria-prima é muito expressiva (Tabela 3).

Considerando-se as características de torração, além de não se ter observado diferença de cor entre denominações (Tabela 2), pode-se verificar que, na média, os teores de compostos sensíveis à torração (ácido nicotínico, trigonelina e 5-ACQ) se mostraram muito semelhantes, independentemente do café ser denominado Forte ou Extra Forte, em que várias amostras foram descritas pelos fabricantes como apresentando torra escura (Tabelas 4 e 5). Esses valores também pouco diferiram da média dos produtos denominados Tradicional, apesar da descrição dos fabricantes indicar torra média (Tabela 3). Esse fato pode ser observado, por exemplo, para as marcas D, F, H, Q e V que tinham produtos tanto na denominação Tradicional (Tabela 3) como Extra Forte (Tabela 5), e a marca E, que apresentava, ainda, produto com denominação Forte (Tabela 4).

Deve-se considerar também que outros fatores podem afetar a concentração dos componentes. Tanto a adição de materiais diferenciados, tais como, milho, trigo, centeio e cevada, ${ }^{46}$ quanto a presença de café com defeitos poderiam implicar em diferença na composição do produto. A adição de um adulterante, ou seja, um produto diferente de café conilon, faria com que os compostos analisados no presente estudo diminuíssem de concentração. Foi relatado que a presença de grãos defeituosos não alterou os teores de diterpenos, ${ }^{16}$ porém, ocorreu aumento nas concentrações de ácidos clorogênicos ${ }^{47,48}$ e trigonelina, ${ }^{49}$ e diminuição nos teores de cafeína, lipídios e polifenóis. ${ }^{20,48}$ No entanto, observou-se, por exemplo, que algumas amostras que tiveram caveol muito baixo (KT e OEF) não apresentaram, necessariamente, redução tão expressiva no teor de cafeína (Tabelas 3 e 5), indicando que, mesmo que exista possibilidade 
de adulteração e/ou presença de defeitos, a espécie empregada continua sendo um dos fatores mais relevantes para a composição.

\section{CONCLUSÃO}

A heterogeneidade observada nos teores dos compostos bioativos entre produtos com diferentes denominações (Tradicional, Gourmet, Forte, Extra Forte, Premium, Aralto, Exportação e Espresso) foi atribuída a diferenças no processo de torração e à matéria-prima utilizada. No entanto, a avaliação de cor e dos teores dos compostos sensíveis à temperatura (ácido nicotínico, trigonelina e 5-ACQ) indicou que poderia haver menos diferença no grau de torra do que o esperado pelas declarações dos fabricantes na embalagem. Considerou-se que a variabilidade nos teores de cafeína e diterpenos (caveol e cafestol), componentes pouco influenciados pelas condições de torração, poderia ser explicada, principalmente, pelas espécies de café (arábica e conilon) empregadas nas formulações dos blends. Cafés da categoria Gourmet caracterizaram-se pelas altas concentrações de diterpenos, trigonelina e 5-ACQ e baixos teores de cafeína, indicativos da grande proporção de café arábica.

\section{MATERIAL SUPLEMENTAR}

No material suplementar, disponível gratuitamente em http:// quimicanova.sbq.org.br, na forma de arquivo PDF, encontram-se as Figuras 1S e 2S.

\section{AGRADECIMENTOS}

Ao $\mathrm{CNPq}$ e ao CBP\&D/Café, pelo apoio financeiro.

\section{REFERÊNCIAS}

1. Trugo, L. C. Em Encyclopedia of Food Sciences and Nutrition; Caballero, B.; Trugo, L. C.; Finglas, P. M., orgs.; Academic Press: London, 2003, vol. 3, p. 1498.

2. Carvalho, L. M.; Silva, E. A. M.; Azevedo, A. A.; Mosquim, P. R.; Cecon, P. R.; Pesq. Agropec. Bras. 2001, 36, 411; http://www.ico.org, acessada em Janeiro 2009.

3. Bragança, S. M.; Carvalho, C. H. S.; Fonseca, A. F. A.; Ferrão, R. G.; Pesq. Agropec. Bras. 2001, 36, 765.

4. Carvalho, V. D. Em Cafeicultura empresarial: produtividade e qualidade; Mendes, A. N. G.; Guimarães, R. G., eds.; UFLA/FAEPE: Lavras, 1998, p. 1.

5. De Maria, C. A. B.; Moreira, R. F. A.; Quim. Nova 2004, 27, 586.

6. França, A. S.; Oliveira, L. S.; Borges, M. L.; Vitorino, M. D.; R. Bras. Armazen. 2001, Especial Café, 37.

7. Ky, C. L.; Louarn, J.; Dussert, S.; Guyot, B.; Hamon, S.; Noirot, M.; Food Chem. 2001, 75, 223.

8. Speer, K.; Kölling-Speer, I.; Braz. J. Plant Physiol. 2006, 18, 201.

9. http://www.abic.com.br, acessada em Janeiro 2009.

10. http://www22.sede.embrapa.br/cafe/outros/links.htm, acessada em Janeiro 2009.

11. http://extranet.agricultura.gov.br/sislegis-consulta/consultarLegislacao. do?operacao=visualizar\&id=18524, acessada em Janeiro 2009.

12. http://www.abic.com.br/serv_legislacao.html, acessada em Janeiro 2009.

13. González, A. G.; Pablos, F.; Martín, M. J.; León-Camacho, M.; Valdenebro, M. S.; Food Chem. 2001, 73, 93; Martín, M. J.; Pablos, F.; González, A. G.; Talanta 1998, 46, 1259.

14. Rubayiza, A. B.; Meurens, M.; J. Agric. Food Chem. 2005, 53, 4654.

15. Higdon, J. V.; Frei, B.; Crit. Rev. Food Sci. Nutr. 2006, 46, 101.

16. Campanha, F. G.; Dias, R. C. E.; Benassi, M. T.; Coffee Science 2010, 5 , no prelo.

17. Dias, R. C. E.; Dissertação de Mestrado, Universidade Estadual de
Londrina, Brasil, 2005.

18. Monteiro, M. C.; Trugo, L. C.; Quim. Nova 2005, 28, 637.

19. Oosterveld, A.; Voragen, A. G. J.; Schols, H. A.; Carbohydr. Polym. 2003, 54, 183.

20. França, A. S.; Mendonça, J. C. F.; Oliveira, S. D.; LWT - Food Sci. Technol. 2005, 38, 709.

21. Moreira, R. F. A.; Trugo, L. C.; De Maria, C. A. B.; Quim. Nova 2000, 23, 195.

22. Farah, A.; De Paulis, T.; Trugo, L. C.; Martin, P. R.; J. Agric. Food Chem. 2005, 53, 1505.

23. Nogueira, M.; Trugo, L. C.; Ciên. Tecnol. Aliment. 2003, 23, 296.

24. Perrone, D.; Farah, A.; Donangelo, C. M.; De Paulis, T.; Martin, P. R.; Food Chem. 2008, 106, 859.

25. Fujioka, K.; Shibamoto, T.; Food Chem. 2008, 106, 217.

26. Perrone, D.; Donangelo, C. M.; Farah, A.; Food Chem. 2008, 110, 1030.

27. http://www.byk.com/instruments/products/color_solidcolor_spectroguide_2_US.php, acessada em Janeiro 2009.

28. Alves, S. T.; Dias, R. C. E.; Benassi, M. T.; Scholz, M. B. S.; Quim. Nova 2006, 29, 1164

29. Dias, R. C. E.; Campanha, F. G.; Vieira, L. G. E.; Ferreira, L. P.; Pot, D.; Marraccini, P.; Benassi, M. T.; J. Agric. Food Chem. 2010, 58, 88.

30. Duarte, G.; Pereira, A. A.; Farah, A.; Food Chem. 2009, $118,851$.

31. STATSOFT 7.1; Statistica for Windows: Computer program manual; Software Inc., Tulsa, 2006.

32. Moura, S. C. S. R.; Germer, S. P. M.; Anjos, V. D. A.; Mori, E. E. M.; Mattoso, L. H. C.; Firmino, A.; Nascimento, C. J. F.; Braz. J. Food Technol. 2007a, 10, 271.

33. Fernandes, S. M.; Pereira, R. G. F. A.; Thé, P. M. P.; Pinto, N. A. V. D.; Carvalho, V. D.; Revista Brasileira de Agrociência 2001, 7, 197; Moura, S. C. S. R.; Germer, S. P. M.; Anjos, V. D. A.; Mori, E. E. M.; Mattoso, L. H. C.; Firmino, A.; Nascimento, C. J. F.; Braz. J. Food Technol. 2007b, 10, 17.

34. Clarke, R. J.; Macrae, R.; Coffee: chemistry, Elsevier: London, 1989.

35. Daglia, M.; Cuzzoni, M. T.; Decano, C.; J. Agric. Food Chem. 1994, 42, 2273.

36. De Maria, C. A. B.; Moreira, R. F. A.; Trugo, L. C.; Quim. Nova 1999, 22, 209; Minamisawa, M.; Yoshida, S.; Takai, N.; Anal. Sci. 2004, 20 , 325.

37. Casal, S.; Oliveira, M. B.; Ferreira, M. A.; Food Chem. 2000, 68, 481.

38. Trugo, L. C.; Macrae, R.; Food Chem. 1984, 15, 219.

39. Casal, S.; Oliveira, M. B. P. P.; Alves, M. R.; Ferreira, M. A.; J. Agric. Food Chem. 2000, 48, 3420.

40. Araújo, J. M. A.; Sandi, D.; Food Chem. 2007, 101, 1087.

41. Frega, N.; Bocci, F.; Lercker, G.; J. High Resol. Chromatogr. 1994, 17, 303; Lercker, G.; Frega, N.; Bocci, F.; Rodriguez-Estrada, M. T.; Chromatographia 1995, 41, 29.

42. Kurzrock, T.; Speer, K.; Food Rev. Int. 2001, 17, 433.

43. Urgert, R.; van der Weg, G.; Kosmeijer-Schuil, T. G.; van de Bovenkamp, P.; Hovenier, R.; Katan, M. B.; J. Agric. Food Chem. 1995, 43, 2167.

44. Nackunstz, B.; Maier, H. G.; Z. Lebensm. Unters. Forsch. 1987, 184 , 494.

45. Lago, R. C. A.; Boletim do CEPPA 2001, 19, 319; Lercker, G.; Caboni, M. F.; Bertacco, G.; Turchetto, E.; Lucci, A.; Bortolomeazzi, R.; Pagani, E.; Frega, N.; Bocci, F.; Industrie Alimentari 1996, 35, 1057.

46. Amboni, R. D. M. C.; Francisco, A.; Teixeira, E.; Ciênc. Tecnol. Aliment. 1999, 19, 311; Jham, G. N.; Berhow, M. A.; Manthey, L. K.; Palmquist, D. A.; Vaughn, S. F.; J. Braz. Chem. Soc. 2008, 19, 1462.

47. Farah, A.; Donangelo, C. M.; Braz. J. Plant Physiol. 2006, 18, 23.

48. Ramalakshmi, K.; Kubra, I. R.; Rao, L. J. M.; J. Food Sci. 2007, 72, S333.

49. França, A. S.; Oliveira, L. S.; Mendonça, J. C. F.; Silva, X. A.; Food Chem. 2005, 90, 89. 


\section{TEORES DE COMPOSTOS BIOATIVOS EM CAFÉS TORRADOS E MOÍDOS COMERCIAIS}

Romilaine Mansano Nicolau de Souza, Gisele André Baptista Canuto, Rafael Carlos Eloy Dias e Marta de Toledo Benassi* Departamento de Ciência e Tecnologia de Alimentos, Universidade Estadual de Londrina, CP 6001, 86051-970 Londrina - PR, Brasil

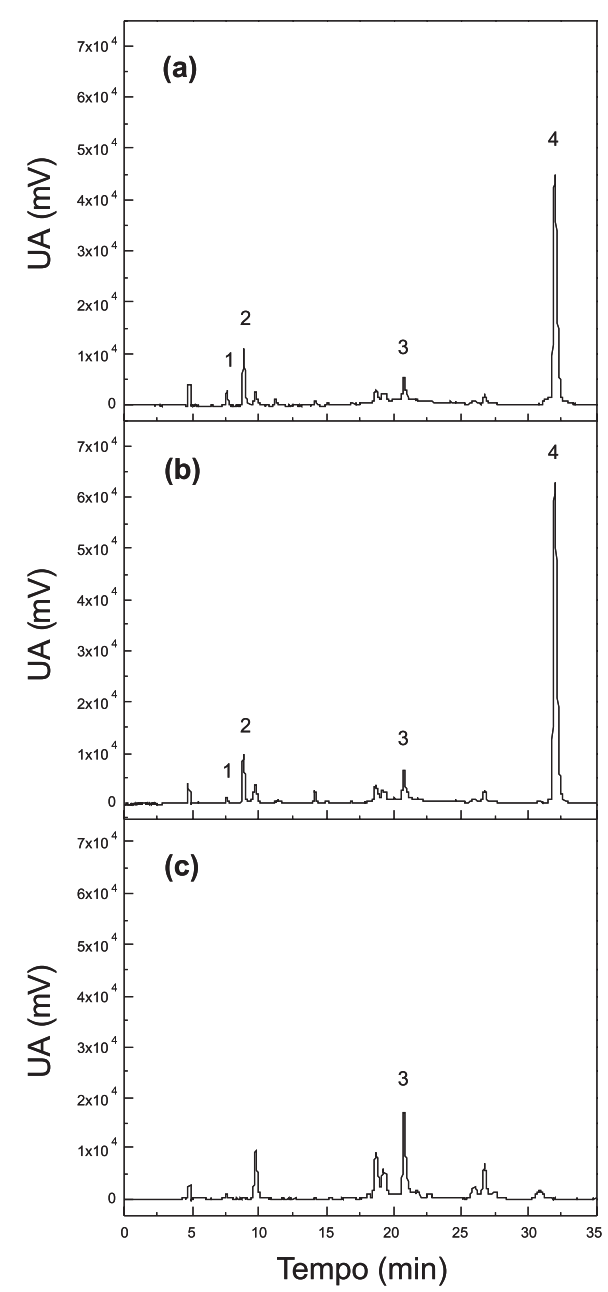

Figura 1S. Cromatogramas típicos dos compostos hidrossolúveis em cafés torrados e moídos comerciais. Detecção de ácido nicotínico e trigonelina a 260 (a), cafeína a 272 (b) e 5-ACQ a $320 \mathrm{~nm}$ (c). Picos: ácido nicotínico (1), trigonelina (2), 5-ACQ (3) e cafeina (4). Condições: coluna Spherisorb ODS-1, $250 \times 4,6$ mm, $5 \mu \mathrm{m}$; gradiente de ác. acético $5 \%$ (A) e acetonitrila (B) $(0$ a 5 ' $-5 \%$ de B; 5' a 10' 5 a 13\% de B, linear; 10' a 35'-13\% de B), vazão de 0,7 mL/min

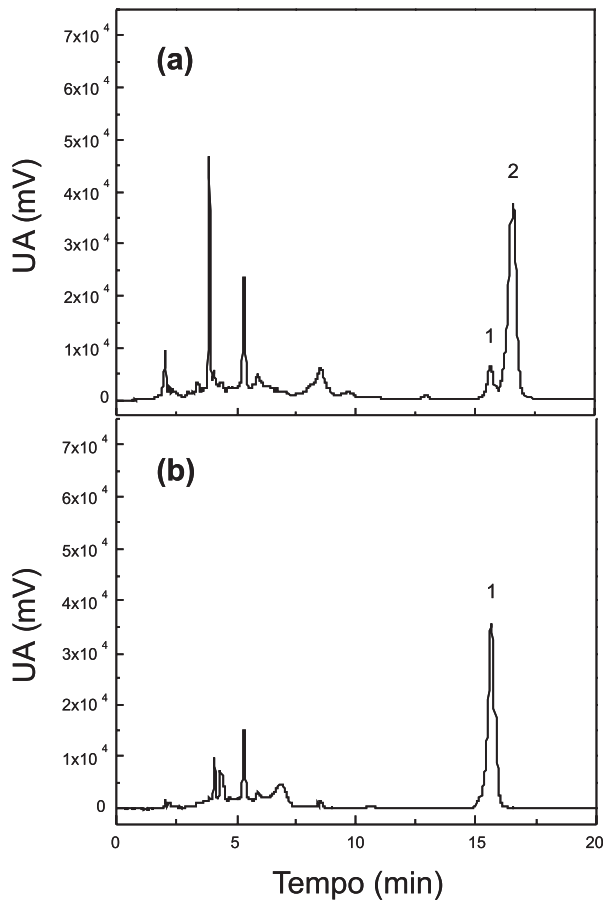

Figura 2S. Cromatogramas típicos dos compostos lipossolúveis em cafés torrados e moídos comerciais. Detecção de cafestol a 230 (a) e caveol a $290 \mathrm{~nm}$ (b). Picos de caveol (1) e cafestol (2). Condições: coluna Spherisorb ODS-1, 250 × 4,6 mm, 5 нm; eluição isocrática com acetonitrila:água (55:45, $v / v)$, vazão de $0,9 \mathrm{~mL} / \mathrm{min}$ 